\title{
Marker antibody expression stratifies Crohn's disease into immunologically homogeneous subgroups with distinct clinical characteristics
}

\author{
E A Vasiliauskas, L Y Kam, L C Karp, J Gaiennie, H Yang, S R Targan
}

\begin{abstract}
Background-Perinuclear antineutrophil cytoplasmic antibodies (pANCA) have been detected in a clinically distinct Crohn's disease subpopulation. Antibodies to Saccharomyces cerevisiae (ASCA) have been demonstrated in the majority of patients with Crohn's disease.

Aims-To examine the relationship between selective marker antibody expression in Crohn's disease and disease onset, location, and clinical behaviour patterns. Methods-Sera from 156 consecutive patients with established Crohn's disease were evaluated in a blinded fashion for the presence of ASCA and ANCA. Clinical profiles were generated by investigators blinded to immune marker status.
\end{abstract}

Results-Using multiple regression analyses, higher ASCA levels were shown to be independently associated with early age of disease onset as well as both fibrostenosing and internal penetrating disease behaviours. Higher ANCA levels were associated with later age of onset and ulcerative colitis-like behaviour. Substratification of the Crohn's disease population using selective ANCA and ASCA expression (high levels of a single marker antibody): (1) distinguished homogeneous subgroups that manifested similar disease location and behaviours; and (2) identified patients with more aggressive small bowel disease.

Conclusions-The findings suggest that by taking into account the magnitude of the host immune response, Crohn's disease can now be stratified on an immunological basis into more homogeneous clinically distinct subgroups, characterised by greater uniformity among anatomical distribution of disease and disease behaviour.

(Gut 2000;47:487-496)

Keywords: antineutrophil cytoplasmic antibody; anti-Saccharomyces cerevisiae antibody; Crohn's disease; inflammatory bowel disease; ulcerative colitis

Correspondence to: Dr E A Vasiliauskas or Dr S $R$ Targan, Inflammatory Bowel Disease Center, Cedars-Sinai Medical Center, $8631 \mathrm{~W}$ Third Street Suite 430E, Los Angeles, CA 90048, USA. Email:

Eric.A.Vasiliauskas@cshs.org or Stephan.Targan@cshs.org

Accepted for publication 12 April 2000

Many variations in the clinical presentation and natural history of Crohn's disease (CD) have been recognised since the original descriptions in the early part of this century. ${ }^{1}$ What is classically termed "Crohn's disease" may actually represent a heterogeneous group of diseases manifesting similar features. ${ }^{2-5}$ Numerous attempts have been made to charac- terise CD patients into uniform subgroups to better understand and predict clinical courses and responses to medical and surgical interventions. To date, most stratification analyses have focused on the anatomical distribution of inflammation and fibrostenosing or perforating/fistulising behaviour patterns. Such classifications are descriptive and have not been shown to reflect specific pathogenic mechanisms.

Serum immune markers have been used to characterise subgroups of patients with ulcerative colitis (UC) and CD. Antineutrophil cytoplasmic antibodies (ANCA), autoantibodies directed against intracellular components of neutrophils, are present in the majority of UC patients and in a clinically distinct CD subpopulation. ${ }^{6}$ ANCA expression in UC permits stratification at the mucosal, clinical, and genetic levels, and has been associated with distinct disease subgroups. ${ }^{7-14}$ UC associated ANCA exhibit perinuclear highlighting (pANCA) on indirect immunofluorescent (IIF) microscopy. The pANCA of UC loses the characteristic perinuclear IIF staining pattern when pretreated with DNase. ${ }^{15}$ Serum pANCA is thought to reflect mucosal pANCA production, and studies have shown that B cell priming and pANCA production take place in the colonic mucosa. These findings imply that recognition of mucosal antigen(s) leads to local production of pANCA. ${ }^{7}$ In relatively small studies, pANCA in UC has been associated with the clinical features of: (1) treatment resistant left sided disease ${ }^{8}$; (2) aggressive disease course $\mathrm{e}^{910}$; (3) surgery early in the disease course $^{11}$; and (4) development of pouchitis following ileal pouch-anal anastomosis. ${ }^{12-14}$ Genetic studies have suggested that subpopulations of UC can be defined by ANCA expression in association with specific HLA markers. ${ }^{16-18}$ In UC, pANCA production may reflect a distinct mucosal inflammatory process and thereby represents immunologically distinct subpopulations.

pANCA expression in CD provides additional evidence that pANCA is a marker of a distinct mucosal inflammatory process. CD
Abbreviations used in this paper: ASCA, anti-Saccharomyces cerevisiae antibody; CD, Crohn's disease; ELISA, enzyme linked immunosorbent assay; EU, ELISA units; IIF, indirect immunofluorescent; IBD, inflammatory bowel disease; pANCA, perinuclear antineutrophil cytoplasmic antibodies; UC, ulcerative colitis; TNF, tumour necrosis factor; PNPP, p-nitrophenol phosphate; Ig, immunoglobulin. 
patients expressing pANCA have clinical features of left sided colitis and endoscopic and/or histopathological features typical of UC. ${ }^{6}$ This "UC-like" phenotype has been confirmed in several studies. ${ }^{18-20}$ Additionally, the serum immunoglobulin $\mathrm{G}$ (IgG) subclass profile of pANCA $^{+}$CD is similar to that of UC. ${ }^{21}$ Thus in $\mathrm{CD}$ the presence of serum pANCA suggests a mucosal inflammatory process in common with the UC pANCA subgroup.

Antibodies to Saccharomyces cerevisiae (ASCA) have been detected in up to $79 \%$ of CD patients. ${ }^{22}$ Expression of this marker antibody may also reflect a specific mucosal immune mediated response. Sendid et al demonstrated that yeast cell wall phosphopeptidomannans were the epitopes responsible for the antigenic reactivity in $\mathrm{ASCA}^{+} \mathrm{CD}$ sera. ${ }^{23} \mathrm{Man}-$ nans are believed to be the major antigenic component of yeast cell walls and are an important antigenic constituent of mycobacteria and other microorganisms. ${ }^{24}$ The clinical significance of ASCA expression in CD is not well defined. However, Darroch et al demonstrated that soluble preparations of $S$ cerevisiae can induce a lymphoproliferative response in vitro, similar to that of known recall antigens. ${ }^{25}$ Taylor et al described the association of ASCA with the tumour necrosis factor (TNF) microsatellite haplotype a11b4c1d3e 3 in patients with inflammatory bowel disease (IBD). ${ }^{26}$ These findings suggest that a gene related to ASCA expression resides on chromosome 6 within the major histocompatibility complex in the vicinity of this haplotype. A mutation located on the haplotype may be contributing to ASCA expression. Taken together, these findings raise the possibility that ASCA expression, in common with pANCA, may reflect specific mucosal immune mechanisms.

Selective marker antibody expression has been used to differentiate between UC and CD, but ASCA and ANCA have not been used to stratify CD subgroups. ${ }^{17}{ }^{27}$ The aim of this study was to examine the association between clinical characteristics and ASCA and/or pANCA expression in CD patients. The results demonstrated that in this referral centre cohort, higher ASCA levels were associated with earlier age of disease onset of CD and both fibrostenosing and internal penetrating disease behaviours. In contrast, higher ANCA levels were associated with an older age of disease onset and a UC-like inflammatory response. Therefore, subgroups of CD patients stratified based on age of onset and clinical characteristics may represent immunologically distinct groups.

\section{Methods}

STUDY POPULATION

The study population consisted of 156 consecutive patients with an established diagnosis of CD evaluated at the CSMC IBD Center, who consented to donate serum for research purposes. This study was reviewed and approved for human subject participation by the Cedars-Sinai Institutional Review Board. Patients with primary sclerosing cholangitis, autoimmune hepatitis, and those with chron- ically elevated transaminases or alkaline phosphatase were excluded to avoid confusion with non-IBD ANCA. ${ }^{28}{ }^{29}$ Diagnosis of CD was defined by the presence of a combination of established features from at least two of the following categories: (1) clinical-perforating or fistulising disease, obstructive symptoms secondary to small bowel stenosis or stricture; (2) endoscopic-deep linear or serpiginous ulcerations, discrete ulcers in normal appearing mucosa, cobblestoning, discontinuous or asymmetric inflammation; (3) radiographicsegmental disease (skip lesions), small bowel or colon strictures, stenosis, or fistula; and/or (4) histopathological-submucosal or transmural inflammation, multiple granulomas, marked focal cryptitis or focal chronic inflammatory infiltration within and between biopsies, or skip lesions including rectal sparing in the absence of local therapy.

ASSESSMENT OF CLINICAL CHARACTERISTICS Clinical assessment and serum immune marker evaluations were performed independently and before data analysis. Clinical information was collected by chart review and patient interview by clinical investigators blinded to individual patient ANCA and ASCA status. Detailed patient profiles were generated using the database intake forms currently in use at the CSMC IBD Center. Epidemiological data included: age, age of IBD symptom onset, disease duration, sex, ethnicity, and family history of CD or UC. Signs and symptoms associated with active CD were noted, including: obstructive symptoms, diarrhoea, bleeding and mucus discharge, urgency, tenesmus, aphthous ulcerations, perianal abscesses or fistulae, anal fissures or strictures, as well as extraintestinal manifestations of IBD. For each patient, the specific anatomical location of all documented areas of endoscopic, surgical, histopathological, or radiographic inflammation, stricturing, fistulisation, perforation, or abscess formation were recorded. Location of disease was further grouped into the standard categories of "small bowel disease only," "small bowel and colonic disease," and "colonic involvement only." Clinical behaviour patterns were characterised as fibrostenotic, penetrating, and/or "UC-like." To be classified as having "fibrostenotic disease" patients needed appropriate radiographic, endoscopic, surgical, or histopathological documentation. Patients with CD related fistulae, abscesses, or perforations were described as having "penetrating disease" and were further substratified into "internal" or "perianal" penetrating disease. CD related fistulae to the urinary tract and to the skin (other than perianal) were considered internal. Vaginal fistulae communicating with the rectum were grouped with perianal fistulae. Fistulae, abscesses, or perforations resulting from surgical complications were not included as "penetrating disease." CD patients were considered "UC-like" if they exhibited clinical features of left sided colonic disease and endoscopic and/or histopathological features of UC as previously described. ${ }^{6}$ Because it is recognised that 
CD behaviour patterns are not mutually exclusive and can coexist within a given individual, a single patient may thus be represented in more than one behaviour analysis. The number, types, and reason for all IBD related surgeries were recorded. Pharmacological interventions were grouped to reflect the use of sulfasalazine or oral 5-aminosalicylic acid derivatives, immunomodulatory agents (6-mercaptopurine, azathioprine, methotrexate, mycophenolate, cyclosporin, FK506, anti-TNF- $\alpha$, interleukin10), IBD directed antibiotic therapy, and topical therapies for distal colonic disease (enemas, foams, or suppositories).

SERUM ANCA DETERMINATION AND ANCA

SUBTYPE CHARACTERISATION

Serum ANCA expression and ANCA subtype characterisation were performed in a blinded fashion by Prometheus Laboratories (San Diego, California, USA). ANCA presence was determined by fixed neutrophil enzyme linked immunosorbent assay (ELISA) as previously described. ${ }^{30}$ Polystyrene microtitre plates were coated with $2.5 \times 10^{5}$ normal human donor whole peripheral blood neutrophils per well, which were fixed with $100 \%$ methanol. Cells were incubated with bovine serum albumin $(0.25 \%)$ in phosphate buffered saline to block non-specific antibody binding. Next, control and coded sera were added at a 1:100 dilution. Following incubation and washing, goat $\mathrm{F}\left(\mathrm{ab}^{\prime}\right)_{2}$ antihuman immunoglobulin $\mathrm{G}$ ( $\gamma$ chain specific) antibody (Jackson Immunoresearch Labs, Inc., West Grove, Pennsylvania, USA) conjugated to alkaline phosphatase was added at a 1:1000 dilution to label neutrophil bound antibody. Substrate solution containing $p$-nitrophenol phosphate (PNPP) was then added. Colour development was allowed to proceed until absorbance at $405 \mathrm{~nm}$ in the positive control wells was $0.8-1.0$ optical density units greater than that in blank wells, using a reference wavelength of $650 \mathrm{~nm}$ on a Molecular Devices (Menlo Park, California, USA) E-Max microtitre plate reader. Levels were determined relative to a Prometheus laboratory standard consisting of pooled sera obtained from well characterised $\mathrm{pANCA}^{+} \mathrm{UC}$ patients. Results were expressed as ELISA units $(\mathrm{EU} / \mathrm{ml})$. Sera with circulating antineutrophil cytoplasmic IgG antibody exceeding the normal reference range value were termed "ANCA ${ }^{+}$." Numeric values below the normal reference range were termed "ANCA"

$\mathrm{ANCA}^{+}$sera were further subtyped via IIF staining to determine the ANCA neutrophil binding pattern. Glass slides containing approximately $10^{5}$ normal human donor whole peripheral blood neutrophils per slide were prepared by cytocentrifugation (Shandon Cytospin, Cheshire, UK). Cells were fixed in $100 \%$ methanol, air dried, and stored at $-20^{\circ} \mathrm{C}$. The coded patient sera were diluted $(1: 20)$ and then layered over the fixed neutrophils, incubated at $37^{\circ} \mathrm{C}$, and washed. Fluorescein labelled $\mathrm{F}\left(\mathrm{ab}^{\prime}\right)_{2} \gamma$ chain specific goat antihuman antibody was added and incubated and washed, as previously described. ${ }^{30}$ The sections were examined by fluorescence microscopy using an epifluorescence equipped Nikon microscope (Garden City, New Jersey, USA). Sera exhibiting the characteristic perinuclear highlighting that then lost this characteristic staining pattern when first treated with DNase were termed "pANCA" ${ }^{+}{ }^{15}$

DETERMINATION AND CHARACTERISATION OF SERUM ASCA

Sera were analysed for ASCA expression in a blinded manner by Prometheus Laboratories using a fixed ELISA assay. High binding polystyrene microtitre plates were coated with purified phosphopeptidomannans extracted from yeast Saccharomyces uvarum, a subspecies of $S$ cerevisiae. Coded patient sera were diluted and added to the wells. Following incubation and washing, alkaline phosphatase conjugated goat antihuman $\mathrm{F}\left(\mathrm{ab}^{\prime}\right)$ fragment specific IgG (Pierce, Rockford, Illinois, USA) or alpha chain specific IgA (Jackson) was added. After incubation, wells were washed, and PNPP substrate was added. Colour development was proportional to concentrations of antibody present in sera. Samples were read at $405 \mathrm{~nm}$ with a reference wavelength of $650 \mathrm{~nm}$ on a Molecular Devices E-Max microtitre plate reader. Levels were determined and results expressed as ELISA units $(\mathrm{EU} / \mathrm{ml})$ relative to a Prometheus laboratory standard which was derived from a pool of patient sera with well characterised $\mathrm{CD}$ found to have reactivity to this antigen. Sera exhibiting ASCA reactivity (IgG and/or IgA) exceeding the normal reference range were termed "ASCA positive" $\left(\mathrm{ASCA}^{+}\right)$.

\section{STATISTICAL ANALYSIS}

Statistical analyses were performed using SAS computer software (Release 6.10; SAS Institute, Inc., Cary, North Carolina, USA, 1994). Both quantitative ASCA and ANCA - that is, antibody levels - and qualitative ASCA and ANCA - that is, antibody positivity and ANCA pattern -were used in analyses. Logarithmic transformation was performed for ASCA and ANCA levels (including those below the reference range) to obtain a more ready normal distribution before correlation was computed and statistical tests performed. Actual numeric values were used when analysing serum immune marker levels as quantitative variables. In practice, ASCA positivity was determined by either IgG or IgA being positive. Similarly, to obtain one measure for a quantitative ASCA level, we used both IgG and IgA values by selecting the larger one of the two standardised values (IgG' or IgA'), where $\operatorname{IgG}{ }^{\prime}=[\operatorname{lnIgG}-$ mean $(\ln I g G)] / S D(\ln I g G)$ and $\operatorname{IgA} A^{\prime}=[\ln I g A-$ mean $(\ln I g A)] / S D(\ln I g A)$. Correlations between normalised variables were evaluated by Pearson correlation coefficients and corresponding $\mathrm{p}$ values. To evaluate the relationship between serum ANCA and ASCA and clinical features, linear multiple regression analysis was performed by specifying antibody levels as dependent variables and clinical characteristics as independent variables using a stepwise procedure. To further corroborate the independent variables identified as significant 
by multiple regression analysis, an additional analysis was performed examining those populations expressing high levels of a single marker antibody. Because there was a significant overlap of ANCA and ASCA, levels $\geqslant 50 \mathrm{EU} / \mathrm{ml}$ were chosen for ASCA and levels $\geqslant 40 \mathrm{EU} / \mathrm{ml}$ for ANCA to allow for a sufficient number of patients for analysis while eliminating patients expressing only low levels of this marker. To test mean differences in normalised quantitative variables between any two groups, the Student's $t$ test was used; between multiple groups, one way ANOVA was used followed by pairwise Fisher's least significant difference test for means. $p$ values for such pairwise tests were adjusted for multiple comparisons. The nonparametric Kruskal-Wallis test was used to compare numbers of small bowel surgeries between groups.

\section{Results}

ANCA EXPRESSION AND IMMUNOFLUORESCENCE STAINING PATTERNS IN CD PATIENTS

In this study, using a well defined CD population and optimised technology, ELISA screening detected serum ANCA above the reference range in $56 \%(87 / 156)$ of patients. Forty one per cent (36/87) of CD patients expressing ANCA exhibited a perinuclear highlighting pattern (pANCA) on IIF staining. To further characterise the pANCA of CD patients, all $\mathrm{pANCA}^{+} \mathrm{CD}$ sera were analysed by pretreatment of neutrophils with DNase and reassessed for the resulting ANCA IIF staining pattern. Complete loss of antigen recognition following treatment with DNase $(\mathrm{P} \rightarrow \varnothing)$ was observed in $78 \%(28 / 36)$ of pANCA $^{+}$samples. Fourteen per cent (5/36) converted to a homogeneous cytoplasmic staining pattern $(\mathrm{P} \rightarrow \mathrm{C})$. Three of $36(8 \%)$ retained the perinuclear highlighting pattern $(\mathrm{P} \rightarrow \mathrm{P})$. The proportion of DNase sensitive pANCA CD serum samples converting from $\mathrm{P} \rightarrow \varnothing$ and from $\mathrm{P} \rightarrow \mathrm{C}$ was similar to that seen in the pANCA ${ }^{+} \mathrm{UC}_{\text {popu- }}$ lation $(70 \%$ and $30 \%),{ }^{15}$ and provides evidence that pANCA of CD is the same as pANCA of UC.

The reported prevalence of ANCA expression in CD has varied greatly due to differences in study populations and detection methodology. In most other reports of ANCA in IBD, ANCA expression was detected by IIF alone. In this study, ANCA expression was determined by ELISA, with assessment of neutrophil staining pattern by IIF, and finally confirmation by DNase digestion of neutrophils. Therefore, in this paper all subsequent references to "pANCA" should be interpreted as DNase sensitive pANCA with loss of antigen recognition following pretreatment with DNase $(\mathrm{P} \rightarrow \varnothing$ or $\mathrm{P} \rightarrow \mathrm{C})$. ELISA levels of ANCA were highest in the pANCA ${ }^{+}$subgroup (mean (SEM): 72 (10) EU/ml) compared with the non-pANCA ${ }^{+}$subgroup (25 (2) EU/ml), and those that were $\mathrm{ANCA}^{-}$(10 (1) $\mathrm{EU} / \mathrm{ml}$ ) $\left(\mathrm{p}_{\chi^{2} \text { for trend }}<0.0001\right)$.

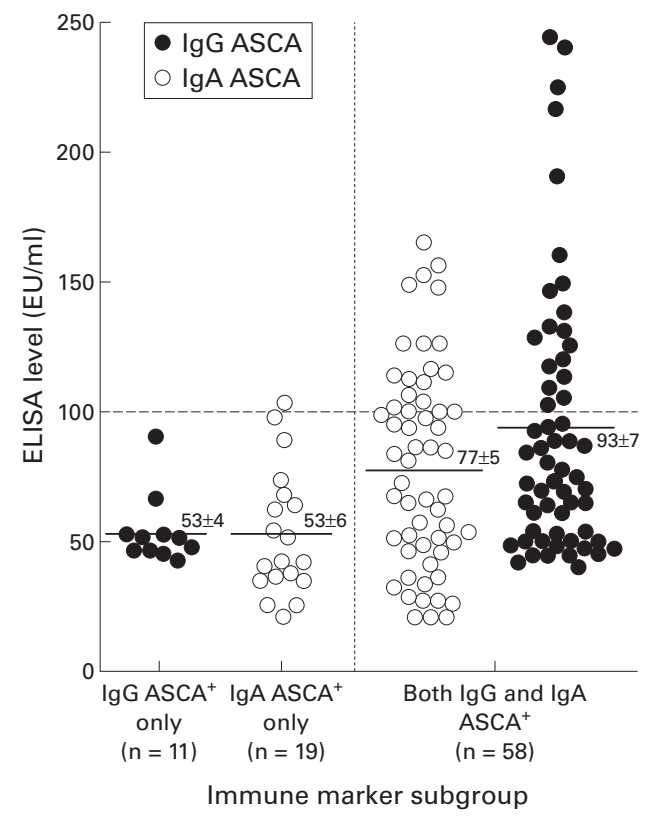

Figure 1 Levels of anti-Saccharomyces cerevisiae antibody (ASCA) positive sera from the Crohn's disease (CD) population. The solid line in each group represents the mean of the subgroup. Almost all patients (25/26) expressing the highest levels of either IgG or IgA ASCA ( $\geqslant 100 \mathrm{EU} / \mathrm{ml}$, values above the broken line) were part of the CD subgroup expressing both IgG and IgA ASCA.

IgG AND/OR IgA ASCA EXPRESSION DISTINGUISHES CD SUBGROUPS

Using a purified antigen preparation and an ELISA optimised for antibody detection, IgG and IgA serum antibodies to Saccharomyces cerevisiae (ASCA) were detected above the reference range in $56 \%(88 / 156)$ of the CD study population. Of the $\mathrm{ASCA}^{+}$patients, $13 \%$ (11/88) expressed IgG ASCA alone, 22\% (19/ 88) expressed IgA ASCA alone, and 66\% (58/ 88) expressed IgG and IgA ASCA. Patients with both IgG and IgA ASCA had higher mean (SEM) quantitative levels (IgG ASCA 93 (7) $\mathrm{EU} / \mathrm{ml}$; IgA ASCA 77 (5) EU/ml) than patients expressing exclusively IgG ASCA (53 (4) $\mathrm{EU} / \mathrm{ml})(\mathrm{p}=0.039)$ or IgA ASCA (53 (6) $\mathrm{EU} / \mathrm{ml}) \quad(\mathrm{p}=0.10) \quad($ fig 1$)$. Figure 1 also illustrates that almost all patients (25/26) expressing the highest levels of either IgG or IgA ASCA ( $\geqslant 100 \mathrm{EU} / \mathrm{ml})$ were part of the CD subgroup expressing both IgG and IgA ASCA.

RELATIONSHIP AMONG MARKER ANTIBODY LEVELS As there was great variation in levels of marker antibody expression among CD patients, we examined the relationship between levels of marker antibody expression. There was a significant positive correlation between IgG ASCA and IgA ASCA levels $(r=0.72$, $\mathrm{p}=0.0001)$ and a negative correlation between IgA ASCA and ANCA levels $(r=-0.21$, $\mathrm{p}=0.009)$. The correlation between IgG ASCA and ANCA levels was also negative $(r=-0.13)$ but did not attain statistical significance $(p=0.11)$. Thus an inverse relationship between ANCA and ASCA levels within this CD patient cohort was suggested (that is, the higher the ASCA level, the lower the ANCA level). This inverse relationship suggests that 
Table 1 Demographic features of the Crohn's disease study population stratified by ANCA and ASCA expression

\begin{tabular}{|c|c|c|c|c|c|c|}
\hline & \multirow[b]{2}{*}{ All $C D$} & \multicolumn{2}{|l|}{$A S C A$} & \multicolumn{3}{|l|}{$A N C A$} \\
\hline & & $A S C A^{+}$ & $A S C A^{-}$ & $p A N C A^{+}$ & Other $A N C A^{+}$ & $A N C A^{-}$ \\
\hline $\mathrm{n}$ & 156 & 88 & 68 & 33 & 54 & 69 \\
\hline $\operatorname{Sex}(M: F)$ & $85: 71$ & $51: 37$ & $34: 34$ & $15: 18$ & $28: 26$ & $42: 27$ \\
\hline Age $(y)$ & $41(1)$ & $38(1)^{\star}$ & $44(2)$ & $40(2)$ & $42(2)$ & $40(2)$ \\
\hline Age at CD onset (y) & $25(1)$ & $22(1)^{\star}$ & $29(2)$ & $26(2)^{\star \star}$ & $27(2)^{\star \star \star}$ & $23(1)$ \\
\hline Duration of disease (y) & $16(1)$ & $17(1)^{\star}$ & $14(1)$ & $14(2)^{\star \star}$ & $14(1)^{\star \star \star}$ & $17(1)$ \\
\hline Jewish ancestry $(\%)$ & $64(41 \%)$ & $40(45 \%)$ & $24(35 \%)$ & $16(48 \%)$ & $22(41 \%)$ & $26(38 \%)$ \\
\hline \multicolumn{7}{|l|}{ Family history } \\
\hline IBD $(\%)$ & $48(31 \%)$ & $28(32 \%)$ & $20(29 \%)$ & $10(30 \%)$ & $16(30 \%)$ & $22(32 \%)$ \\
\hline $\mathrm{CD}(\%)$ & $33(21 \%)$ & $19(22 \%)$ & $14(21 \%)$ & $6(18 \%)$ & $13(24 \%)$ & $14(20 \%)$ \\
\hline UC $(\%)$ & $17(11 \%)$ & $9(10 \%)$ & $8(12 \%)$ & $2(6 \%)$ & $7(13 \%)$ & $8(12 \%)$ \\
\hline Immunomodulatory therapy (\%) & $126(81 \%)$ & $77(88 \%)^{\star}$ & $49(72 \%)$ & $27(82 \%)$ & $39(72 \%)$ & $60(87 \%)$ \\
\hline
\end{tabular}

Values are mean (SEM) or number (\%).

ASCA, anti-Saccharomyces cerevisiae antibody; pANCA, perinuclear antineutrophil cytoplasmic antibodies; CD, Crohn's disease; IBD, inflammatory bowel disease; UC, ulcerative colitis.

${ }^{\star} \mathrm{p}_{\mathrm{t} \text {-test }}<0.0001, \mathrm{ASCA}^{+} v \mathrm{ASCA}^{-} ;{ }^{\star \star} \mathrm{p}_{\text {Fisher's LSD test from ANOVA }}<0.0001, \mathrm{pANCA}^{+} v \mathrm{ANCA}^{-} ;{ }^{\star \star \star} \mathrm{p}_{\text {Fisher's LSD test from ANOVA }}<0.0001$, other $\mathrm{ANCA}^{+} v \mathrm{ANCA}^{-}$.

the presence and levels of a specific marker antibody could reflect distinct immunological reactivity leading to differences in clinical expression among CD patients.

\section{RELATIONSHIP BETWEEN MARKER ANTIBODY EXPRESSION AND LEVEL AND DEMOGRAPHIC CHARACTERISTICS WITHIN CD PATIENTS}

Multiple investigations have demonstrated that serum and genetic markers can be correlated with identifiable CD phenotypes. ${ }^{6}{ }^{18-20} 27$ 31-35 The demographic variables of sex, current age, Jewish ancestry, and family history of CD, UC, or IBD in general, were compared between antibody marker subgroups (table 1). Except for age, there were no significant differences either between $\mathrm{ASCA}^{+}$and $\mathrm{ASCA}^{-}$subgroups or among $\mathrm{pANCA}^{+}$, other $\mathrm{ANCA}^{+}$, and $\mathrm{ANCA}^{-}$subgroups. Although more $\mathrm{ASCA}^{+}$ patients $(88 \%)$ had been treated with immunosuppressive therapies than those that were $\operatorname{ASCA}^{-}(72 \%)(p<0.0001)$, this referral centre cohort as a whole had severe disease, as reflected by the use of immunosuppressive interventions in $81 \%$ of patients overall.

\section{Age of onset}

The majority of epidemiological studies examining age of presentation suggest a bimodal age distribution in $\mathrm{CD}^{36-39}$ To begin to assess whether clinical differences between childhood and adult onset CD in disease distribution and clinical course ${ }^{40-47}$ may be associated with different immune responses, we examined the relationship between age of onset of $\mathrm{CD}$ and expression of these immune markers. Patients in whom ASCA was detected experienced onset of IBD symptoms significantly earlier in life than patients not expressing ASCA (mean

Table 2 Age at onset of Crohn's disease stratified by ANCA and ASCA expression

\begin{tabular}{cclll}
\hline & $\begin{array}{l}\text { Pre-teenage onset CD } \\
(<13 y) \text { (range 4-12 } \\
y)(n=22)\end{array}$ & $\begin{array}{l}\text { Teenage and young } \\
\text { adult onset CD (range } \\
13-40 y)(n=110)\end{array}$ & $\begin{array}{l}\text { Late adult onset } \\
\text { CD ( }>40) \text { (range } \\
42-67 y)(n=24)\end{array}$ & $p$ Value \\
\hline $\begin{array}{c}\mathrm{ASCA}^{+} \\
\text {IgG and IgA }\end{array}$ & $16(73 \%)$ & $65(59 \%)$ & $7(29 \%)$ & 0.003 \\
$\mathrm{ASCA}^{+}$ & $11(50 \%)$ & $43(39 \%)$ & $4(17 \%)$ & 0.02 \\
$\mathrm{ANCA}^{+}$ & $12(55 \%)$ & $58(53 \%)$ & $17(71 \%)$ & $\mathrm{NS}$ \\
pANCA $^{+}$ & $4(18 \%)$ & $23(21 \%)$ & $6(25 \%)$ & $\mathrm{NS}$ \\
\hline
\end{tabular}

Statistical differences in proportions were evaluated using the $\chi^{2}$ test for trend.

ASCA, anti-Saccharomyces cerevisiae antibody; ANCA, antineutrophil cytoplasmic antibodies; CD, Crohn's disease.
(SEM) 22 (1) $v 29$ (2) years, respectively) $(\mathrm{p}<0.0001)$ (table 1$)$, corroborating previous observations by Quinton and colleagues. ${ }^{27}$ In fact in a multivariate analysis of ASCA levels (see methods) there was a significant correlation with early age of onset $(p<0.005)$. As current age is not independent of patient's age at disease onset and/or duration of disease, we examined these three age related factors together using multiple regression analysis to evaluate which factor had the primary association with levels of IgG or IgA ASCA. Age of onset was shown to be independently associated with levels of both IgG and IgA ASCA but not current age or duration of disease. The negative correlation between IgG and $\operatorname{IgA}$ ASCA levels and age of onset of CD (IgG $r=-0.33, \mathrm{p}=0.0001$; IgA $r=-0.30, \mathrm{p}=0.0002$ ) provides further evidence for the association of this immune response with younger age of onset. The effect of age of onset on expression of ASCA, and ANCA expression and levels in patients with pre-teenage (age $<13$ years) onset of CD was compared with marker expression in patients with late adult onset (age $>40$ years) CD (age $>40$ has been described as the start of the second peak age for onset of CD).$^{36-39}$ As depicted in table 2,73\% (16/22) of patients with pre-teenage onset of CD were $\mathrm{ASCA}^{+}$ compared with $59 \%(65 / 110)$ of patients with onset between 13 and 40 years, and only $29 \%$ $(7 / 24)$ of patients with disease onset after age 40 years $\left(\mathrm{p}_{x^{2} \text { for trend }}<0.003\right)$. Although the per cent of CD patients expressing pANCA did not vary significantly with age of onset, there was a tendency towards higher levels of ANCA expression with increasing age of onset $(p=0.019)$. Early and late onset CD may have distinct immunological responses.

RELATIONSHIP BETWEEN MAGNITUDE OF MARKER ANTIBODY EXPRESSION AND CLINICAL FEATURES OF CD

The variability of levels of ASCA (fig 1) and ANCA expression and the inverse relationship of these marker antibodies within this cohort led to analysis of the relationship between levels and clinical features. ASCA (IgG or IgA) and ANCA were measured as a continuous trait (that is, levels). To increase the statistical power and evaluate the relationship between 
Table 3 Clinical features: results of multivariate analysis

\begin{tabular}{|c|c|c|c|c|c|}
\hline & $\begin{array}{l}\text { Disease } \\
\text { location }\end{array}$ & $\begin{array}{l}\text { Fibrostenosing } \\
\text { disease }\end{array}$ & $\begin{array}{l}\text { Internal penetrating } \\
\text { disease }\end{array}$ & $\begin{array}{l}\text { Perianal penetrating } \\
\text { disease }\end{array}$ & $\begin{array}{l}\text { UC-like } \\
\text { features }\end{array}$ \\
\hline \multicolumn{6}{|l|}{ ASCA level } \\
\hline$\beta$ & - & 0.33 & 0.32 & - & -0.29 \\
\hline$r^{2}$ & - & 0.13 & 0.03 & - & 0.02 \\
\hline p Value & NS & 0.0001 & 0.022 & NS & 0.063 \\
\hline \multicolumn{6}{|l|}{ ANCA level } \\
\hline$\beta$ & - & - & - & - & 0.67 \\
\hline$r^{2}$ & - & - & - & - & 0.15 \\
\hline $\mathrm{p}$ Value & NS & NS & NS & NS & 0.0001 \\
\hline
\end{tabular}

$\beta$, regression coefficient; $r^{2}$, proportion of the total variation of a dependent variable can be explained by the variation of each independent variable.

ASCA, anti-Saccharomyces cerevisiae antibody; ANCA, antineutrophil cytoplasmic antibodies.

antibody expression and clinical features of CD in an objective manner, we first conducted a multiple regression analysis using the quantitative ASCA or ANCA level as a dependent variable and all clinical features as independent variables, including anatomical distribution of disease and disease behaviours (fibrostenosing, internal penetrating, perianal penetrating, and UC-like).

Table 3 lists the clinical features that showed significant association with either ASCA or ANCA. As overall ASCA positivity was determined by the presence of either the $\operatorname{IgG}$ or IgA ASCA isotype, we used both IgG and IgA values by selecting the larger one of the two standardised values (as described in methods). Higher levels of ASCA were associated with fibrostenosing disease, internal penetrating disease, and less often UC-like features (table 3). In contrast, higher levels of ANCA levels were associated with UC-like features only (table 3).

The presence of each of these serum immune markers has been associated with disease location. ${ }^{6} 1819273132$ In this cohort, the presence of pANCA was associated with colonic involvement $(88 \% ; 29 / 33)$ and ASCA with small bowel involvement (alone or in combination with colonic disease) $(90 \% ; 79 / 88)$. Ileal involvement was seen in all 79 patients; $93 \%$ $(64 / 69)$ and $90 \%(69 / 77)$ of IgG $\mathrm{ASCA}^{+}$and IgA ASCA ${ }^{+}$, respectively. However, when levels of these serum immune markers were analysed with respect to disease location, there was no independent association.

SUBSTRATIFICATION USING SELECTED EXPRESSION OF IMMUNE MARKERS FURTHER CHARACTERISES DISTINCT CLINICAL PHENOTYPES IN CD

In this study the associations with behavioural manifestations in CD became more evident at higher levels of both marker antibodies. Higher levels of ASCA expression were associated with the traditional "classic" fibrostenosing and internal fistulising features of $\mathrm{CD}$, while higher levels of ANCA were associated with UC-like features. This demonstrates that not only the type, but also the magnitude of the immune marker expression (as measured by level of host marker antibody production) may reflect divergent immune responses that manifest as specific disease behaviours (phenotypes) in these subsets of CD patients.

To further evaluate this hypothesis, we examined those populations expressing high levels of a single marker antibody, that is: (1)

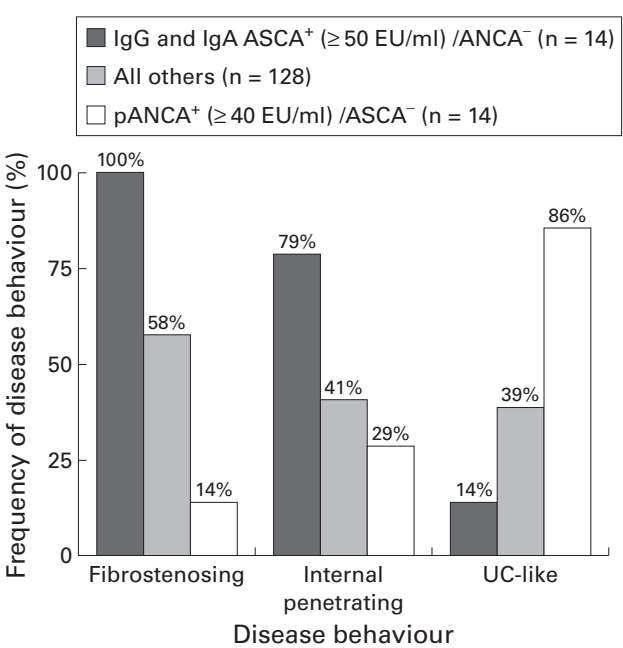

Figure 2 Substratification using selected expression of immune markers. Disease behaviour characteristics were examined in more immunologically homogeneous subgroups of patients with Crohn's disease (CD) (those expressing high levels of a single marker antibody) and compared with all other CD study patients. Overall differences in proportions were evaluated using the $\chi^{2}$ test for trend ( $p<0.001$ for each of the disease behaviour characteristics).

high levels of ASCA without ANCA (IgA and $\operatorname{IgG~ASCA}{ }^{+} \geqslant 50 \mathrm{EU} / \mathrm{ml}$ and $\mathrm{ANCA}^{-}$); and (2) high levels of pANCA without ASCA $\left(\mathrm{pANCA}^{+} \geqslant 40 \mathrm{EU} / \mathrm{ml}\right.$ and $\left.\mathrm{ASCA}^{-}\right)$. Because there was a significant overlap of ANCA and ASCA, levels $\geqslant 50 \mathrm{EU} / \mathrm{ml}$ were chosen for ASCA to allow for a sufficient number of patients to be analysed while eliminating patients expressing only low levels of this marker. As illustrated in fig 2, all patients with IgA and IgG $\mathrm{ASCA}^{+} \geqslant 50 \mathrm{EU} / \mathrm{ml}$ and the $\mathrm{ANCA}^{-}$immune marker subprofile had developed fibrostenosis $(14 / 14 ; 100 \%)$. Most experienced internal penetrating complications (11/ $14 ; 79 \%)$; only $14 \%(2 / 14)$ had UC-like features. In contrast, $86 \%(12 / 14)$ of $\mathrm{pANCA}^{+}$ $\geqslant 40 \mathrm{EU} / \mathrm{ml}$ and $\mathrm{ASCA}^{-} \mathrm{CD}$ patients exhibited UC-like features while only $14 \%(2 / 14)$ of patients in this subgroup had fibrostenotic disease and $29 \%(4 / 14)$ had internal penetrating disease. Fibrostenosing and internal penetrating behaviours have been considered by some to be indicators of a more aggressive form of small bowel CD. ${ }^{48-54}$

The need for small bowel surgery or recurrent surgeries has been suggested as a further measure of disease severity. To take into account the observation that the incidence of small bowel disease varied between immune marker subgroups, we examined prevalence and numbers of surgeries per patient among those with small bowel involvement (alone or in combination with colonic disease). Analysis revealed that small bowel surgery was required by $86 \%(12 / 14)$ of the IgA and IgG $\mathrm{ASCA}^{+}$ $\geqslant 50 \mathrm{EU} / \mathrm{ml}$ and $\mathrm{ANCA}^{-}$subgroup (fig $3 \mathrm{~A}$ ). This was significantly more than the pANCA ${ }^{+}$ $\geqslant 40 \mathrm{EU} / \mathrm{ml}$ and $\mathrm{ASCA}^{-}$group $(2 / 7 ; 29 \%)$ and the remainder of the study patients with small bowel involvement $(56 / 99 ; 57 \%)(p=0.0001)$. Additionally, the mean number of small bowel surgeries per patient with small bowel disease was higher in the IgA and IgG $\mathrm{ASCA}^{+} \geqslant 50$ 

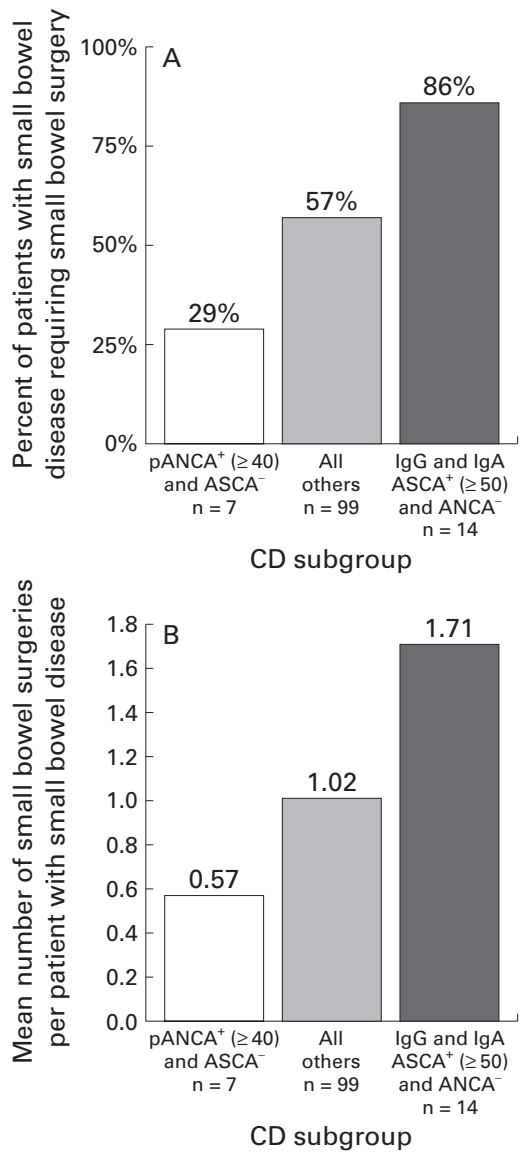

Figure 3 Surgery as a measure of disease aggressiveness. Substratification of the subset of patients with small bowel Substratification of the subset of patients with small bowel
disease (Crohn's disease (CD) involving the small bowel alone or in combination with the colon) using selective immune marker expression. (A) Percentage of patients requiring small bowel surgery and $(B)$ total number of small bowel surgeries per patient with small bowel involvement. Overall differences in proportions were evaluated using the $\chi^{2}$ test for trend. $(A: p<0.0001 ; B$ : $p<0.005)$.

$\mathrm{EU} / \mathrm{ml}$ and $\mathrm{ANCA}^{-}$(1.71/patient) subgroup than in the $\mathrm{pANCA}^{+} \geqslant 40 \mathrm{EU} / \mathrm{ml}$ and $\mathrm{ASCA}^{-}$ subgroup (0.57/patient) and all others (1.02/ patient) $(p=0.0054)$ (fig 3B). Therefore, higher levels of ASCA without ANCA may reflect more aggressive small intestinal disease.

\section{Discussion}

We have demonstrated that serum ASCA and ANCA status are criteria by which patients with $\mathrm{CD}$ can be stratified into distinct clinical subgroups. Levels of ASCA were independently associated with earlier age of onset of CD and a tendency towards developing classic fibrostenosing and internal penetrating small bowel complications (table 3). In contrast, higher levels of pANCA were associated with later age of onset and a UC-like inflammatory response, as well as a relative lack of fibrostenosis and penetrating disease (table 3). Finally, the concept of substratifying the CD population using selective ANCA and ASCA expression was demonstrated as a complementary approach for identification of immunologically and clinically homogeneous subgroups (fig 2), including a group with a more aggressive disease course (figs 2, 3A, 3B). Most studies that have examined expression of marker antibodies in IBD have focused on the presence or absence of the particular antibody and the presence or absence of what we currently term "UC" or "CD". 61819232730 Our study revealed that the presence of these marker antibodies and the magnitude of the host immune response correlated with distinct, and indeed divergent, clinical characteristics, and suggests that ASCA and pANCA are serum markers for different mucosal inflammatory mechanisms that influence disease expression.

Of the patient characteristics evaluated, age of disease onset correlated strongly with disease behaviour and anatomical location. It has been observed that childhood onset CD is characterised by a greater prevalence of small bowel disease and stricturing/penetrating complications compared with late onset which is associated with more colonic disease and a lower incidence of fistula formation. ${ }^{44}$ Other studies suggest that childhood onset $C D$ has a more aggressive course ${ }^{4041}$ while older onset is associated with a comparatively favourable prognosis, better response to medical therapies, and lower risk of recurrence in the minority requiring surgery. ${ }^{39-47}$ The results of our study corroborate these clinical observations. In this CD cohort early disease onset was associated with increased incidence of small bowel involvement and fibrostenotic, as well as internal perforating complications (data not shown). In contrast, CD limited to the colon and UC-like features were more frequent in late onset $\mathrm{CD}$, with the disease course less often complicated by fibrostenosis or internal perforating complications (data not shown). These differences in disease location and behaviour pattern tendencies suggest that paediatric and later adult onset CD represent pathogenetically distinct forms of the disease.

The underlying pathogenetic mechanisms for these clinical differences observed between adult versus childhood onset CD remain unclear. Although familial clustering supports the role of genetic factors in the pathogenesis of paediatric and adult onset CD, additional factors must also be involved to account for the observed differences. ${ }^{55-60}$ It has been hypothesised that young children may be more immunologically reactive than older children or adults. ${ }^{61-63}$ Age dependent immune influences have been described in other immune mediated disorders. ${ }^{64-67}$ The results of this study provide evidence that age of onset associated with host dependent immune responses may influence intestinal location, phenotypic expression, and CD behaviour.

Disease location is another variable previously found to positively correlate with immunological markers such as ASCA and ANCA expression. Giaffer et al observed that patients with isolated small bowel involvement had significantly higher ASCA IgG titres against two strains of $S$ cerevisiae than those with colonic disease. ${ }^{31}$ In a study examining ASCA expression in monozygotic twins, Lindberg et al found higher IgG ASCA levels (to whole yeast) in patients with CD limited to the small bowel compared with those with both 
small bowel and colonic involvement. ${ }^{32}$ In contrast, several investigators have suggested that pANCA expression in CD is related to colonic disease which at minimum is left sided. ${ }^{6}{ }^{18}{ }^{19} \mathrm{In}$ the present study, we extended this observation, describing the importance of considering both pattern and quantitative levels of marker antibody expression when defining this association. Although the majority of $\mathrm{ASCA}^{+}$patients had CD involving the small bowel and most $\mathrm{pANCA}^{+}$patients had colonic involvement, levels of the serum immune markers were not independently associated with disease location.

Stratification based on CD behaviour has been widely studied and reported, often including an "inflammatory" behaviour in the analysis. All CD related behaviours are the result of inflammation, and therefore this variable was not included separately in the stratification analysis in this study. Higher levels of ASCA expression are associated with the well characterised subgroups of patients with the classic complications of fibrostenosing and internal penetrating disease. While perforating and non-perforating behaviours are clinically (and likely in some ways immunologically) distinct also, the observation that higher levels of ASCA expression are associated with both of these traditional "classic" fibrostenosing and internal penetrating features of CD suggests an overlapping mucosal mechanism rather than distinct mechanisms. In contrast, CD patients with higher ANCA levels experience these complications less frequently. Furthermore, with internal penetrating disease, no significant correlation was seen with these markers and perianal fistulising disease. This suggests that these two penetrating forms of CD involve different immunological mechanisms and may be considered not only clinically but immunologically distinct.

UC-like behavioural attributes were recently described as the predominant behaviour pattern common to the subgroup of CD patients expressing serum pANCA. ${ }^{618}{ }^{19}$ This study extends previously reported observations. The incidence of UC-like behaviour was greatest at higher ELISA levels of pANCA expression and by exclusion of ASCA expression. The frequency of UC-like features in low level expression of pANCA was no different than in the overall population, emphasising the importance of taking into account the magnitude of the immune response. In this study sera were screened at 1:100 dilution, which may further explain why some studies that included low level pANCA expression have found less pronounced associations. These data strengthen the assertion that $\mathrm{CD}$ and UC pANCA subgroups share a similar mucosal immune response.

Based on our multiple regression analysis that defined the independently associated variables, ASCA and pANCA expression defined different host immune responses, each of which was associated with a different disease behaviour. Thus stratifying patients by expression of only one or the other marker antibody might better define specific clinical phenotypes. Furthermore, stratification by expression of pANCA or ASCA with the added variable of the magnitude of expression may better define subgroups of CD patients into more homogeneous clinical subsets. The use of this approach was demonstrated in this study. We found that the subset of CD patients that expressed higher levels of both IgG and IgA ASCA and not ANCA experienced a more aggressive course of disease. A precedent has been set in UC, where pANCA expression is associated with a more resistant disease course. $^{8-14}$ The subset of patients that expressed higher levels of both IgG and IgA ASCA and not ANCA may have a more aggressive form of small bowel CD. Evidence in support of this characterisation include: (1) universal occurrence of fibrostenosis; (2) frequent development of internal penetrating complications; (3) observation that within the subset of all patients with small bowel disease a higher percentage of patients required small bowel surgery; and, (4) number of small bowel surgeries was three times higher in the IgA and IgG ASCA ${ }^{+} \geqslant 50 \mathrm{EU} / \mathrm{ml}$ and $\mathrm{ANCA}^{-}$subgroup than the $\mathrm{pANCA}^{+} \geqslant 40 \mathrm{EU} / \mathrm{ml}$ and $\mathrm{ASCA}^{-}$ subgroup.

\section{Conclusions}

Our results demonstrate that Crohn's diseases can now begin to be stratified into distinct subgroups by pairing clinical features with the presence and levels of serum antibody markers. The study demonstrated the significance of taking into account the magnitude of the host immune response - that is, higher levels of ASCA and pANCA expression to identify CD subgroups that are immunologically and clinically more homogeneous. To approach the underlying mechanisms involved in the various forms of CD, clinical and genetic studies of CD must rely on homogeneous patient subgroups. ${ }^{52}$ The use of ASCA and pANCA allows for identification of more homogeneous subgroups of patients with common subclinical and clinical characteristics, based on immune mechanisms of disease. Further corroboration of these results is warranted. In particular, the value of marker antibody determination performed at the time of diagnosis of IBD as a predictor of future disease behaviour expression needs to be evaluated in a prospective fashion. None the less, the immunological responses in IBD, as measured by marker antibody production, may well reflect different host genetic profiles.

Stratification of Crohn's diseases into more uniform subgroups using these and other immune markers may permit better characterisation and prediction of natural history. If these findings are reproduced in a clinically less severe population prospectively, this information could potentially allow for earlier and more aggressive treatment to avoid predictable complications in selected patients, without unnecessarily exposing others to potential adverse side effects of overaggressive therapeutic regimens. In addition, stratification based on markers will filter out the "background noise" inherent in studies of a heterogeneous group of diseases with similar characteristics. Stratified populations will facilitate the 
identification of pathogenically more homogeneous subsets of patients and perhaps identify "at risk individuals" in a group of diseases with complex genetic influences, including reduced penetrance or variable expression of disease.

The authors would like to acknowledge Rachel Kramer for data collection and interpretation. The study was supported by USPHS Program Project Grant DK46763, the Cedars-Sina Inflammatory Bowel Disease Research Education Fund, the Cedars-Sinai Board of Governors' Chair in Medical Genetics.

Conflict of interest: Dr Stephan R Targan is a cofounder and current equity holder of Prometheus Laboratories (San Diego, California, USA)

1 Crohn BB, Ginzburg L, Oppenheimer GD. Regional Ileitis. 7AMA 1932;99:1323-9.

2 Targan S, Murphy LK. Serologic and mucosal markers of ulcerative colitis and Crohn's disease: implications of pathophysiology for diagnosis. In: Scholmerich J, Kruis W, poebell H, et al, eds. Inflammatory bowel diseasespathophysiology as basis of treatment. Dordrecht: Kluwer pathophysiology as basis of treatment.

3 Rotter JI, Yang H. Delineating the major aetiological risk factors for IBD: the genetic susceptibilities. In: Scholm-
erich J, Kruis W, Goebell $\mathrm{H}$, et al, eds. Inflammatory bowel erich J, Kruis W, Goebell $\mathrm{H}$, et al, eds. Inflammatory bowel
diseases-pathophysiology as basis of treatment. Dordrecht: diseases-pathophysiology as basis of treatmen
Kluwer Academic Publishers, 1993:9-18.

4 Satsangi J, Jewell DP, Rosenberg WMC, et al. Genetics of inflammatory bowel disease. Gut 1994;35:696-700.

5 Yang H, Rotter JI. The genetics of inflammatory bowe disease: genetic predispositions, disease markers, and genetic heterogeneity. In: Targan SR, Shanahan F, eds. Inflammatory bowel disease: from bench to bedside. Baltimore: Williams and Wilkins, 1994:32-64.

6 Vasiliauskas EA, Plevy SE, Landers CJ, et al. Perinuclear antineutrophil cytoplasmic antibodies in patients with Crohn's disease define a clinical subgroup. Gastroenterology 1996;110:1810-19.

7 Targan SR, Landers CJ, Cobb L, et al. Perinuclear anti-neutrophil cytoplasmic antibodies are spontaneously produced by mucosal B cells of ulcerative colitis patients. frodumunol 1995;155:3262-7.

8 Sandborn WJ, Landers CJ, Tremaine WJ, et al. Association of antineutrophil cytoplasmic antibodies with resistance to treatment of left-sided ulcerative colitis: results of a pilot study. Mayo Clin Proc 1996;71:431-6.

9 Vecchi M, Bianchi MB, Sinico RA, et al. Antibodies to neutrophil cytoplasm in Italian patients with ulcerative colitis sensitivity, specificity and recognition of putative antigens. Digestion 1994;55:34-9.

10 Vecchi M, Bianchi MB, Calabresi G, et al. Long-term observation of the perinuclear anti-cytoplasmic antibody status in ulcerative colitis patients. Scand $\mathcal{F}$ Gastroenterol 1998;33 $170-3$.

11 Boerr LA, Sambuelli AM, Katz S, et al. Clinical heterogeneity of ulcerative colitis in relation to frequency of pANCA eity of ulcerative colitis in relation to frequ
reactivity. Gastroenterology 1995;108:A785.

12 Vecchi M, Gionchetti P, Bianchi MB, et al. p-ANCA and development of pouchitis in ulcerative colitis patients after proctocolectomy and ileoanal pouch anastomosis. Lancet proctocolectomy

13 Patel RT, Stokes R, Birch C, et al. Influence of total colectomy on serum antineutrophil cytoplasmic antibodies in inflammatory bowel disease. Br F Surg 1994;81:724-6.

14 Sandborn WJ, Landers CJ, Tremaine WJ, et al. Antineutrophil cytoplasmic antibody correlates with chronic pouchitis after ileal pouch-anal anastomosis. $A m \quad \mathcal{F}$ Gastroenterol 1995;90:740-7.

15 Vidrich A, Lee J, James E, Cobb L, et al. Segregation of pANCA antigenic recognition by DNase treatment of neutrophils: ulcerative colitis, type 1 autoimmune hepatitis, and primary sclerosing cholangitis. $\mathcal{F}$ Clin Immunol 1995;15:293-9.

16 Toyoda H, Wang SJ, Yang HY, et al. Distinct associations of HLA class II genes with inflammatory bowel disease. GasHLA class II genes with inflam

17 Yang HY, Rotter JI, Toyoda H, et al. Ulcerative colitis: a genetically heterogeneous disorder defined by genetic
(HLA class II) and subclinical (antineutrophil cytoplasmic (HLA class II) and subclinical (antineutrophil cyto

18 Satsangi J, Landers CJ, Welsh KI, et al. The presence of anti-neutrophil antibodies reflects clinical and genetic heterogeneity within inflammatory bowel disease. Inflamm Bowel Dis 1998;4:18-26.

19 Ruemmele FM, Targan SR, Levy G, et al. Diagnostic accuracy of novel serological assays in pediatric inflammatory bowel disease. Gastroenterology 1998; 115:822-9.

20 Freeman HJ. Atypical perinuclear antineutrophil cytoplasmic antibodies (p-ANCA) in patients with Crohn's disease. Gastroenterology 1998;114:A979.

21 Plevy SE, Landers CJ, Vasiliauskas EA, et al. Alterations in serum immunoglobulin (Ig) $\mathrm{G}$ subclasses provide evidence for distinct immune responses in pANCA positive Crohn's disease patients. Gastroenterology 1996;110:A993.

22 Barclay GR, McKenzie H, Pennington J, et al. The effect of dietary yeast on the activity of stable chronic Crohn's disease. Scand $\mathcal{F}$ Gastroenterol Suppl 1992;27:196-200.
23 Sendid B, Colombel JF, Jacquinot PM, et al. Specific antibody response to oligomannosidic epitopes in Crohn's antibody response to oligomannosidic epitopes in
disease. Clin Diagn Lab Immunol 1996:3:219-26.

24 McKenzie H, Main J, Pennington CR, et al. Antibody to selected strains of Saccharomyces cerevisiae (baker's and brewer's yeast) and Candida albicans in Crohn's disease. Gut 1990;31:536-8.

25 Darroch CJ, Christmas SE, Barnes RM. In vitro human lymphocyte proliferative responses to a glycoprotein of the yeast Saccharomyces cerevisiae. Immunology 1994;81:24752.

26 Taylor KD, Li Z, Barry M, et al. Tumor necrosis factor microsatellite haplotype A11B4C1D3E3 is associated with anti-Saccharomyces cerevisiae antibody (ASCA) across clinical forms of inflammatory bowel disease. Gastroenterology 1998:114:A1098.

27 Quinton JF, Sendid B, Reumaux D, et al. AntiSaccharomyces cerevisiae mannan antibodies combined with antineutrophil cytoplasmic autoantibodies in inflammatory bowel disease: prevalence and diagnostic role. Gut 1998;42: 788-91.

28 Duerr RH, Targan SR, Landers CJ, et al. Neutrophil cytoplasmic antibodies: a link between primary sclerosing cholangitis and ulcerative colitis. Gastroenterology 1991; 100:1385-91.

29 Targan SR, Landers C, Vidrich A, et al. High-titer antineutrophil cytoplasmic antibodies in type-1 autoimmune hepatitis. Gastroenterology 1995;108:1159-66.

30 Saxon A, Shanahan F, Landers C, et al. A distinct subset of antineutrophil cytoplasmic antibodies is associated with inflammatory bowel disease. I Allergy Clin Immunol 1990;86:202-10.

31 Giaffer $\mathrm{MH}$, Clark A, Holdsworth CD. Antibodies to Saccharomyces cerevisiae in patients with Crohn's disease and their possible pathogenic importance. Gut 1992;33: $1071-5$.

32 Lindberg E, Magnusson K-E, Tysk C, et al. Antibody (IgG, IgA, and IgM) to baker's yeast (Saccharomyces cerevisiae), yeast mannan, gliadin, ovalbumin and betalactoglobulin in monozygotic twins with inflammatory bowel disease. Gut 1992;33:909-13.

33 Elmgreen J, Sorenson H, Berkowicz A. Polymorphism in complement $\mathrm{C} 3$ in chronic inflammatory bowel disease. Predominance of the C3F gene in Crohn's disease. Acta Med Scand 1984;2155:375-8.

34 Hesresbach D, Alizadeh M, Bretagne JF, et al. Investigation of the association of major histocompatibility complex genes, including HLA class I, class II and TAP genes, with clinical forms of Crohn's disease. Eur F Immunogenet 1996; 23:141-51.

35 Bouma G, Poen AC, Garcia-Gonzalez MA, et al. HLA$\mathrm{DRB} 1{ }^{\star} 03$, but not the TNFA-308 promoter gene polymorphism, confers protection against fistulising Crohn's disease. Immunogenetics 1998; $47: 451-5$.

36 Norlén BJ, Krause U, Bergman L. An epidemiological study of Crohn's disease. Scand f Gastroenterol Suppl 1970;5:38590

37 Lee FI, Costello FT. The changing incidence of Crohn's disease in Blackpool 1969-1983. Front Gastrointest Res 1986;11:102-13.

38 Halme L, von Smitten K, Husa A. The incidence of Crohn's disease in the Helsinki metropolitan area during 19751985. Ann Chir Gynaecol 1989;78:115-19.

39 Fleischer DE, Grimm IS, Friedman LS. Inflammatory bowel disease in older patients. Med Clin North Am 1994;78:1303-19.

40 Puntis J, McNeish AS, Allan RN. Long term prognosis of Crohn's disease with onset in childhood and adolescence. Gut 1984;25:329-36.

41 Cooke WT. Long term prognosis of Crohn's disease with onset in childhood and adolescence. Gut 1984;25:1303-5.

42 Mashako MN, Cezard JP, Navarro J, et al. Crohn's disease esions in the upper gastrointestinal tract: correlation between clinical, radiological, endoscopic, and histological features in adolescents and children. F Pediatr Gastroenterol Nutr 1989;8:442-6.

43 Lenaerts C, Roy CC, Vaillancourt M, et al. High incidence of upper gastrointestinal tract involvement in children with Crohn's disease. Pediatrics 1989;83:777-81.

44 Polito JM II, Childs B, Mellits ED, et al. Crohn's disease: influence of age at diagnosis on site and clinical type of disease. Gastroenterology 1996;111:580-6.

45 Devlin HB, Datta D, Dellipiani AW. The incidence and prevalence of inflammatory bowel disease in North Tees Health District. World $\mathcal{F}$ Surg 1980;4:183-93.

46 Gupta S, Saverymuttu SH, Keshavarzian A, et al. Is the pattern of inflammatory bowel disease different in the elderly? Age Ageing 1985;14:366-70.

47 O'Keefe EA, Wright JP, Froggatt J, et al. Medium-term follow-up of Crohn's disease in Cape Town. $S$ Afr Med $\mathcal{F}$ 1989;76:139-41.

48 Whelan G, Farmer RG, Fazio VW, et al. Recurrence after surgery in Crohn's disease: relationship to location of disease (clinical pattern) and surgical indication. Gastroenterology 1985;88:1826-33.

49 Farmer RG, Whelan G, Fazio VW. Long-term follow-up of patients with Crohn's disease: relationship between the clinical pattern and prognosis. Gastroenterology $1985 ; \mathbf{8 8}$ : $1818-25$.

50 Greenstein AJ, Lachman P, Sachar DB, et al. Perforating and non-perforating indications for repeated operations in Crohn's disease: evidence for two clinical forms. Gut 1988; 29:588-92. 
51 Rutgeerts P, Geboes K, Vantrappen G, et al. Predictability of the postoperative course of Crohn's disease. Gastroenterol the postoperative course
ogy 1990;99:956-63.

52 Sachar DB, Andrews HA, Farmer RG, et al. Proposed classification of patient subgroups in Crohn's disease. Gastroenterol Int 1992;5:141-54.

53 Aeberhard P, Berchtold W, Riedtmann HJ, et al. Surgical recurrence of perforating and nonperforating Crohn's disease: a study of 101 surgically treated patients. Dis Colon Rectum 1996;39:80-7.

54 Sachar DB, Subramani K, Mauer K, et al. Patterns of postoperative recurrence in fistulizing and stenotic Crohn's
disease: a retrospective cohort study of 71 patients. $\mathcal{F}$ Clin Gastroenterol 1996;22:114-16.

55 Monsen U, Bernell O, Johansson C, et al. Prevalence of inflammatory bowel disease among relatives of patients with Crohn's disease. Scand F Gastroenterol Suppl 1991;26: 302-6.

56 Yang H, McElree C, Roth M-P, et al. Familial empirical risks for inflammatory bowel disease: differences between Jews for inflammatory bowel disease: differ

57 Bayless TM, Tokayer AZ, Polito JM II, et al. Crohn's disease: concordance for site and clinical type in affected family members - potential hereditary influences. Gastroen terology 1996;111:573-9.

58 Polito JM II, Rees RC, Childs B, et al. Preliminary evidence for genetic anticipation in Crohn's disease. Lancet 1996, 347:798-800.
59 Satsangi J, Grootscholten C, Holt $\mathrm{H}$, et al. Clinical patterns of familial inflammatory bowel disease. Gut 1996;38:73841 .

60 Peeters $M$, Nevens $H$, Baert F, et al. Familial aggregation in Crohn's disease: increased age-adjusted risk and concordance in clinical characteristics. Gastroenterology 1996;111: 597-603.

61 Ettenger RB, Blifeld C, Prince H, et al. The pediatric nephrologist's dilemma: growth after renal transplantation and its interaction with age as a possible immunologic variable. f Pediatr 1987;111:10225.

62 Ettenger RB. Children are different: the challenges of peditric renal transplantation. Am f Kidney Dis 1992;20:668-

63 Yanase Y, Tango T, Okumura K, et al. Lymphocyte subsets identified by monoclonal antibodies in healthy children. Pediatr Res 1986;20:1147-51.

64 Tucker LB, Menon S, Schaller JG, et al. Adult- and childhood-onset systemic lupus erythematosus: a comparison of onset, clinical features, serology, and outcome. $B r f$ Rheumatol 1995;34:866-72.

65 Cole GF, Stuart CA. A long perspective on childhood multiple sclerosis. Dev Med Child Neurol 1995;37:661-6.

6 von Mutius E. Progression of allergy and childhood to adolescence. Thorax 1996;51(suppl 1):S3-6.

67 Host A, Jacobsen HP, Halken S, et al. The natural history of cow's milk protein allergy/intolerance. Eur $\mathcal{F}$ Clin Nutr 1995;49(suppl 1):S13-18. 